\title{
Results from HST Observations of Six LMC Globular Cluster Fields
}

\author{
K.A.G. Olsen \\ Cerro Tololo Inter-American Observatory, Casilla 603, La Serena, Chile \\ P.W. Hodge \\ Astronomy Department, Box 351580, University of Washington, Seattle, \\ WA 98195, USA
}

M. Mateo

Department of Astronomy, University of Michigan, 821 Dennison Building, Ann Arbor, $M I$ 48109, USA

E.W. Olszewski

Steward Observatory, University of Arizona, Tucson, AZ 85721, USA

\author{
R.A. Schommer, N.B. Suntzeff, and A.R. Walker \\ Cerro Tololo Inter-American Observatory, Casilla 603, La Serena, Chile
}

\begin{abstract}
We present deep $H S T$ color-magnitude diagrams of fields centered on the six old LMC globular clusters NGC 1754, NGC 1835, NGC 1898, NGC 1916, NGC 2005, and NGC 2019. Separate cluster and field star CMDs are shown. The time of formation of the LMC is studied from an analysis of the cluster CMDs. Based on a comparison of the CMDs with sequences of the Milky Way clusters M3, M5, and M55, we suggest that the LMC formed its first stars at the same time as the Milky Way to within 1 Gyr. We find additional evidence that these LMC globular clusters are as old as the oldest Milky Way clusters through a comparison of our data with the horizontal branch evolutionary models of Lee, Demarque, \& Zinn (1994).

The evolution of the LMC following its formation is studied through an analysis of the field star CMDs. Through an automated comparison with stellar evolution models, we extract the star formation histories implied by the CMDs. Our best-fit star formation histories imply that the LMC has been actively forming stars over the last $4 \mathrm{Gyr}$, in agreement with previous field star studies. The four fields that lie in the Bar also contain significant numbers of stars with ages of 4-8 Gyr in the best-fit cases. The most notable disagreement between the best-fit models and observed CMDs is in the color of the red giant branch.
\end{abstract}




\section{Observations}

Observations of the clusters NGC 1754, NGC 1835, NGC 1898, NGC 1916, NGC 2005, and NGC 2019 were taken with HST during Cycle 5. Each cluster was observed for one orbit with WFPC2 through the F555W and F814W filters.

\section{Analysis}

After performing photometry with DoPHOT 2.5, calibrating, and statistically subtracting field stars from the cluster CMDs, we analyzed the ages of the clusters in two ways. First, we matched the LMC cluster CMDs with fiducial sequences of Milky Way clusters having similar metallicities. Ages of the LMC clusters were then measured relative to those of the Milky Way fiducials from the differences in the colors of their RGBs (cf. VandenBerg, Bolte, \& Stetson 1990). Compared with the Milky Way clusters M3, M5, and M55, we find $\left\langle\right.$ Age $_{\mathrm{LMC}}-$ Age $\left._{\mathrm{MW}}\right\rangle=1.0 \pm 1.3 \mathrm{Gyr}$. Second, we compared the HB morphologies and metallicities measured from the RGBs with the HB evolutionary tracks of Lee, Demarque, \& Zinn (1994). Within the errors, five of the LMC clusters fall on the track associated with the old Milky Way halo. The sixth LMC cluster, NGC 1916, was not analyzed because of its high degree of differential reddening.

Star formation histories were extracted from the field star CMDs by fitting Bertelli et al. (1994) isochrones through a technique similar to that described by Dolphin (1997). For these fits, we adopted the age-metallicity relation suggested by LMC clusters and tested different IMFs, distances, and reddenings. For a Salpeter IMF, we find that the NGC 1754 field, which lies in the LMC disk, has a star formation rate that is low until $4 \mathrm{Gyr}$ ago, after which it increases steadily. The four fields lying in the LMC Bar, however, also show high star formation activity in the age range 4-8 Gyr. As this activity appears unique to the Bar, we suggest that the Bar may be an old feature.

The detailed star formation histories may be incorrect because of some mismatch of the models and observations. We find that the old, metal-poor models are unable to fit both the lower main sequence and RGB simultaneously. This suggests that either the adopted age-metallicity relation is incorrect, in which case the LMC globular clusters studied here are $\sim 0.5$ dex more metal-poor than our measurements, or the model RGBs are too red by $\sim 0.08$ magnitudes. Despite this problem, the large differences in the star formation histories of the NGC 1754 field and the Bar fields should remain.

Acknowledgments. We thank M. Bolte, J. Johnson, and G. Mandushev for their unpublished MW fiducials, and D. VandenBerg for valuable discussion. This work was supported by STScI grant GO-05916 to NBS.

\section{References}

Bertelli, G. et al. 1994, A\&AS, 106, 275

Dolphin, A. 1997, New Astronomy, 2, 397

Lee, Y.-W., Demarque, P., \& Zinn, R. 1994, ApJ, 423, 248

VandenBerg, D., Bolte, M., \& Stetson, P. 1990, AJ, 100, 445 